REVIEW ARTICLE

\author{
E.S. Schwartz \\ D.J. Dlugos \\ P.B. Storm \\ J. Dell \\ R. Magee \\ T.P. Flynn \\ D.M. Zarnow
}

R.A. Zimmerman

T.P.L. Roberts

\section{Magnetoencephalography for Pediatric Epilepsy: How We Do lt}

SUMMARY: Magnetoencephalography (MEG) is increasingly being used in the preoperative evaluation of pediatric patients with epilepsy. The ability to noninvasively localize ictal onset zones (IOZ) and their relationships to eloquent functional cortex allows the pediatric epilepsy team to more accurately assess the likelihood of postoperative seizure freedom, while more precisely prognosticating the potential functional deficits that may be expected from resective surgery. Confirmation of clinically suggested multifocality may result in a recommendation against resective surgery because the probability of seizure freedom will be low. Current paradigms for motor and somatosensory testing are robust. Paradigms allowing localization of those regions necessary for competent language function, though promising, are under continuous optimization. MR imaging white matter trajectory data, created from diffusion tensor imaging obtained in the same setting as the localization brain MR imaging, provide ancillary information regarding connectivity of the IOZ to sites of rapid secondary spread and the spatial relationship of the $\mathrm{IOZ}$ to functionally important white matter bundles, such as the corticospinal tracts. A collaborative effort between neuroradiology, neurology, neurosurgery, neuropsychology, technology, and physics ensures successful implementation of MEG within a pediatric epilepsy program. $\mathbf{0}$ ne in 1000 children is affected by epilepsy annually, with an overall incidence of 1 in 5000. Children with epilepsy have an increased mortality rate and are more likely to have traumatic injury than their peers. ${ }^{1,2}$ Progressive neurologic deterioration may also result from uncontrolled frequent seizures. ${ }^{3-5}$ When seizures cannot be adequately controlled with antiepileptic medication, surgery is considered. Once reserved for only the most severe cases, resective surgery is being increasingly used in children due to the risks of long-term antiepileptic medication use and the risk of developmental delay or frank encephalopathy from uncontrolled seizures. There is the additional potential for benefit of early resective surgery to minimize long-term functional loss, due to the significant brain plasticity in the pediatric patient. ${ }^{6-8}$

Magnetoencephalography (MEG) holds promise as a noninvasive tool to localize the ictal onset zones (IOZ) and functional cortex in pediatric patients with treatment-resistant epilepsy. ${ }^{9-19}$ Through the detection of magnetic fields generated by abnormal interictal electric brain activity, patients who may benefit from surgical resection may be more appropriately selected. MEG localization is particularly valuable when an anatomic lesion cannot be found, even on high-field thin-section volumetric brain MR imaging, ${ }^{20-24}$ as well as when multifocal or diffuse disease is clinically suggested. ${ }^{15,16,22,25-31}$

Our experience with MEG in pediatric patients with epilepsy studied at our institution is reviewed. We compare the localization of the IOZ and functional cortex on the basis of traditional evaluation (including clinical seizure features, interictal electroencephalography [EEG], ictal EEG, MR imaging, nuclear imaging, neuropsychological testing, Wada procedure, and functional cortical mapping) with localization on

From Divisions of Neuroradiology (E.S.S., J.D., R.M., D.M.Z., R.A.Z., T.P.L.R.), Neurology (D.J.D.), Neurosurgery (P.B.S.), and Department of Psychology (T.P.F.), The Children's Hospital of Philadelphia, Philadelphia, Pa.

Please address correspondence to Timothy P.L. Roberts, PhD, CHOP Radiology, Suite 2115, Wood Building, 324 S 34th St, Philadelphia, PA 19104-4399; e-mail: robertstim@ email.chop.edu

Indicates article with supplemental on-line table.

DOI 10.3174/ajnr.A1029
MEG. Our patients represent a typically diverse group referred for consideration of epilepsy surgery with a wide variety of seizure frequency, seizure type, and indications for consideration for seizure surgery. The data provided on the first 10 patients closely reflect our clinical practice and MEG referral pattern (On-line Table) and illustrate the various roles that MEG can play.

\section{Indications}

We emphasize the 4 potential roles for MEG in the localization of the IOZ and functional cortex: 1) MEG confirmation of localization based on traditional noninvasive methods, allowing epilepsy surgery to proceed without long-term intracranial EEG monitoring; 2) MEG demonstration of bilateral, multifocal, or diffuse ictal onset, indicating an unfavorable candidate for epilepsy surgery; 3) MEG localization adjacent to or distant from that of traditional methods or focally, when traditional methods suggest multifocal activity, potentially altering clinical decision-making regarding epilepsy surgery; and 4) MEG localization of eloquent cortex to guide surgical trajectory. These data will serve to refine prospective studies on the optimal use of MEG in the localization of the IOZ and functional cortex in children with treatment-resistant epilepsy.

\section{Technique}

Patient preparation is minimal, and the examination is generally extremely well tolerated. Our MEG technologists orient the children to the biomagnetometer and the functional tasks before beginning the examination, in some instances going so far as to provide the patients with the list of words to be presented in the word recall task days before the examination to increase their confidence and, therefore, their comfort level. In our experience, patients younger than 5 years of age require general anesthesia (GA) to complete the examination successfully (limiting the scope of the examination to the assessment of spontaneous discharges and somatosensory function identification). An anesthetic regimen using propofol and remifentanil has been shown not to interfere significantly with 

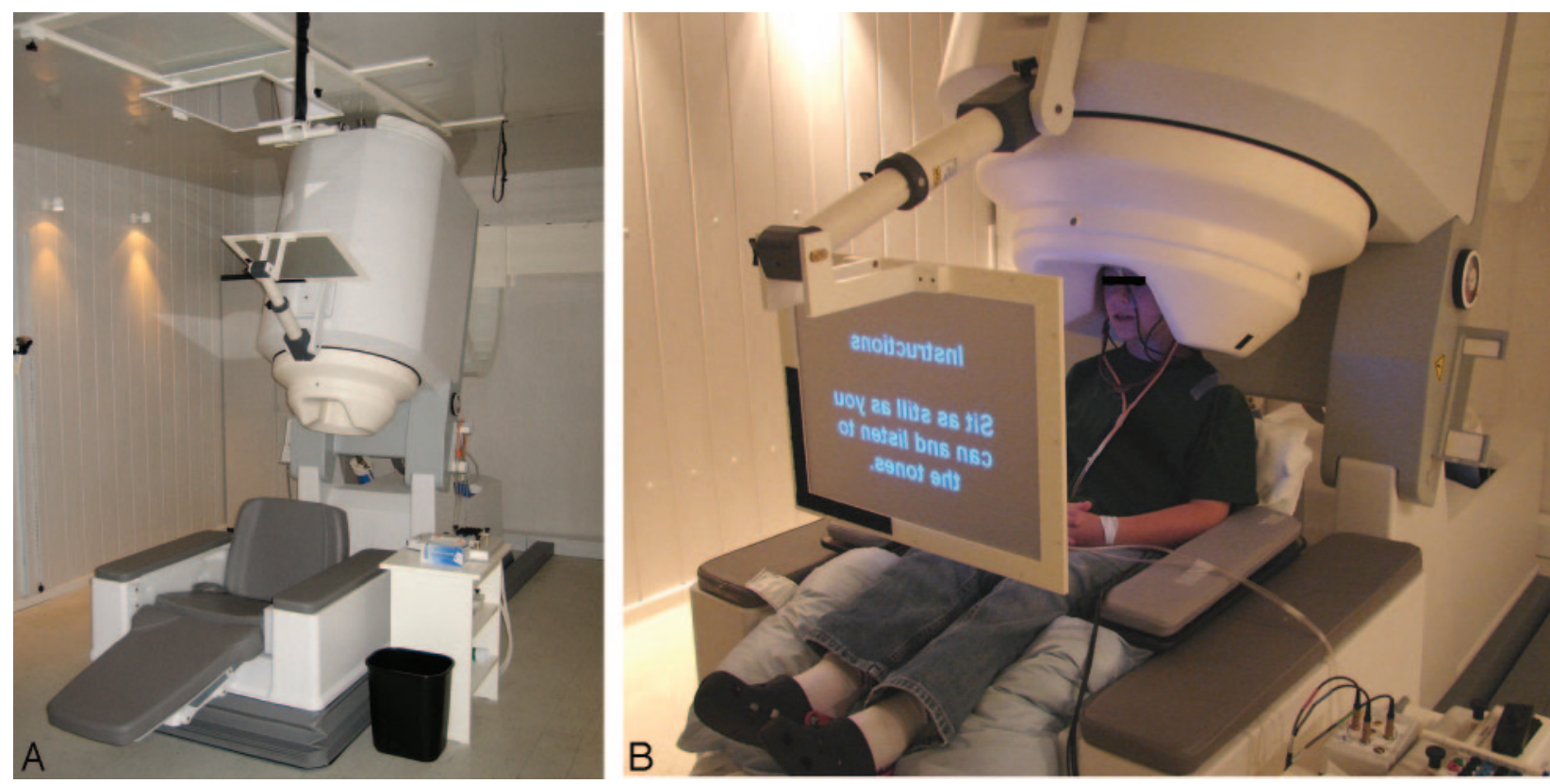

Fig 1. $A$, Biomagnetometer in an electrically shielded room. Patients can be comfortably studied in a seated or supine position. $B$, The video screen is easily viewed and headphones facilitate communication with the technologists and allow auditory stimulus delivery. The wires attached to the 3 electrically active fiducial coils are visible.

these applications of MEG. ${ }^{32,33}$ Older patients with developmental delay may also require GA or anxiolytic administration, and each case is individually assessed by the referring epileptologist and the MEG team.

All studies are performed in a magnetically shielded room by using our 275-channel whole-head biomagnetometer (VSM MedTech, Coquitlam, BC, Canada) (Fig 1). MEG and volumetric MR imaging coregistration are performed along with real-time head motion determination and correction. Three electrically active coils are placed as fiducial markers at the nasion and $1 \mathrm{~cm}$ anterior to the left and right tragus. Activated with currents at frequencies beyond the range of brain activity, these coils generate magnetic fields, which are sampled by the biomagnetometer coils at frequent $(\sim 15 \mathrm{~ms})$ intervals, allowing spatial localization. On the basis of real-time assessment of head position, appropriate transformation can be made to effectively spatially realign different temporal segments of recorded data. Synthetic third-order gradiometer technology is used to reject strong distant sources (such as cardiac electromagnetic activity).

Although we are not generally recording simultaneous EEG, literature indicates that these concurrent data may be valuable in the adult population with epilepsy ${ }^{18,34,35}$ and potentially in the pediatric population. ${ }^{29,36}$ We plan to add this to our routine practice shortly, though determination of tolerance by pediatric patients remains to be established. Clearly, if the application of the leads for the simultaneous EEG recording decreases patient tolerability and increases patient motion during data acquisition or decreases the ability of MEG to detect the IOZ, the potential benefit of simultaneous EEG recording in the pediatric population would be reduced.

After overnight sleep deprivation (to increase interictal activity), MEG recording is performed at a sample rate of 1200 $\mathrm{Hz}$ for fifteen 2-minute recordings, with the patient in the awake, drowsy, and sleeping states to detect interictal MEG sharp waves and spikes, as well as bursts of rhythmic activity.
An automated spatial filtering and spike-detection algorithm detects regions of abnormal transient MEG activity, identified as elevated kurtosis in data filtered with a passband of 20-70 $\mathrm{Hz}$ (SAMg2). ${ }^{37,38}$ Subsequently, time-activity curves from regions of significant activity are examined to identify transient periods of eye-blink, muscular clenching, and other forms of artifact. On elimination of time windows capturing these artifacts, the spatial filtering and spike-detection algorithm is reapplied to the remaining time-domain data. This process is commonly repeated iteratively through 3 or more cycles. Identified regions, meeting appropriate statistical criteria for the presence of elevated kurtosis and showing time-domain evidence of transient sharp activity, are overlaid in color on the MR imaging as statistical probability maps of sharp activity. Virtual or synthetic depth electrodes, constructed by appropriate mathematic weighting of the 275 sensor channel signals, are "placed" at these locations to identify the time-activity profile at these sites and allow morphologic characterization of the regional electric activity.

Subsequent to the passive recording of endogenous electromagnetic activity, MEG recording is also applied to examine responses evoked by stimulation and/or task performance. One or 2 language tests are administered via a video screen placed in front of the patient: picture-naming (via the modified Boston Diagnostic Aphasia Examination) and word recall, to evaluate event-related desynchronization (ERD) in the alpha $(8-15 \mathrm{~Hz})$, beta $(15-30 \mathrm{~Hz})$, and low gamma $(25-50 \mathrm{~Hz})$ bands associated with linguistic computation in a 500-ms latency range immediately before the patient's behavioral response. ${ }^{39}$ Visual assessment of hemispheric differences in ERD is made for frontal and posterior temporal regions (Broca and Wernicke areas, respectively). Some authors have proposed constructing language laterality indices derived from hemispheric differences in the statistical significance (ie, $t$ statistics) of such ERD. ${ }^{39}$

Motor mapping is routinely performed separately with a 


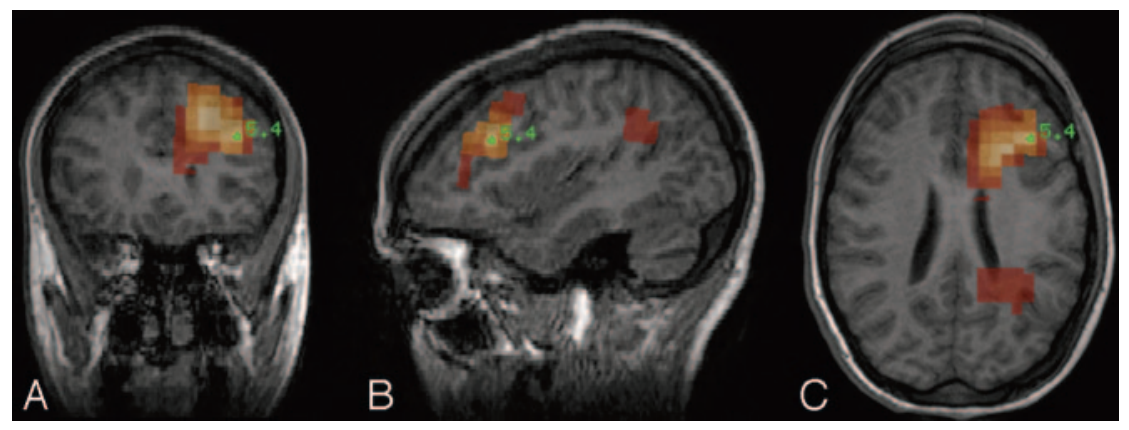

Fig 2. MEG SAMg2 data superimposed on coronal reformation $(A)$, sagittal reformation $(B)$, and axial MPRAGE MR imaging $(C)$. Note the value of the $t$ statistic indicated at the point of peak activity in the region. The statistical map overlay is thresholded to display only pixels with a $t$ statistic value exceeding 2.0 .

self-paced (3-4 second) button-press, by using the left and right index fingers to similarly evaluate ERD in the beta band in the interval $300 \mathrm{~ms}$ before through $200 \mathrm{~ms}$ after the buttonpress. When indicated by the seizure semiology and/or scalp EEG findings, motor mapping can also be performed by using the great toes and elbows.

Somatosensory testing with 3-4 Hz of pneumatic stimulation (air-puff) can be performed on nearly any part of the body. We routinely stimulate the tips of the right and left index fingers and perform more detailed somatosensory mapping (eg, of the face) when indicated. Painless electric stimulation of the right and left median nerves is performed in cases in which the patient is under GA. Electrical stimulation of the median nerve via 500 pulses, each of 0.2-ms duration, at a voltage level just sufficient to elicit thumb motion, is applied at a rate of approximately 3 per second, with a jittered interstimulus interval.

Whole brain MR imaging is performed on our standard clinical $1.5 \mathrm{~T}$ or $3 \mathrm{~T}$ systems, depending on clinical scanner availability. A volumetric T1-weighted magnetization-preparation rapid acquisition of gradient echo (MPRAGE) sequence is acquired in the axial plane, with $0.9-$ to $1.5-\mathrm{mm}$ section thickness. Diffusion tensor imaging (DTI), with application of tensor gradients in at least 6 directions, is also performed in the axial plane with 2 - to 3 -mm contiguous sections, by using an echo-planar acquisition. White matter trajectories are subsequently generated from this DTI data by using the fiber assignment by continuous tracking algorithm to track the white matter trajectory via the step-wise principal eigenvector of the voxel diffusion tensor. ${ }^{40,41}$ The addition of the MR trajectory data allows determination of the relationship of the IOZ to functionally significant white matter tracts, such as the corticospinal tracts, and aids in the identification of white matter pathways that allow rapid propagation of abnormal activity from the IOZ.

\section{Case Examples}

A typical case is that of a 19-year-old profoundly retarded right-handed girl, who had refractory epilepsy that began at 3 months of age. Despite an extensive noninvasive work-up, it is unclear whether her seizures were left frontal or bifrontal in onset. Her clinical seizures were of 3 types: infrequent generalized tonic clonic seizures; frequent (multiple times per day) myoclonic jerks of both upper extremities; and infrequent partial seizures with right-arm extension, head turning to the right, followed by a secondary generalized tonic-clonic seizure. Her EEG findings were abnormal, with a slow background and left frontal or bifrontal sharp waves. Ictal EEG recorded 1 seizure with left frontal lobe onset before rapid bilateral progression and 5 nonlateralized frontal lobe sei(SPECT) favored a left-sided focus, with the ictal scan favoring a left temporal abnormality, whereas the interictal scan showed left temporal, left parietal, and left basal ganglia abnormalities. Wada testing was not possible due to her cognitive dysfunction.

MEG showed transient frequent spike activity, which localized predominantly to the medial left frontal lobe at the junction of the superior frontal and middle frontal gyri and (to a lesser degree) in the left posterior temporal-parietal lobe junction near the region of the angular gyrus. Activity consistently localized to these locations in the left hemisphere (Fig 2).

In the setting of nonlesional MR imaging and bifrontal EEG abnormalities before MEG, the patient was not deemed a surgical candidate. The MEG findings (specifically of a single major IOZ) prompted phase 2 evaluation, consisting of leftsided subdural EEG grid placement and long-term monitoring with capture of multiple typical seizures. The intracranial monitoring confirmed the MEG findings and led to a left temporal lobectomy and focal left inferior frontal lobe resection.

The patient had 1 prolonged seizure 1 month after surgery but has been seizure-free since ( 12 months). Additionally, she has benefited from an improvement in her verbal language ability.

In a contrary scenario, a 4-year-old boy without definite hand preference had refractory atonic seizures characterized by sudden loss of neck tone, sometimes with falling. He was known to have right temporal lobe cortical dysplasia visible on MR imaging. Interictal EEG had shown various patterns, including a normal background with right temporal sharp waves and irregularly generalized sharp waves, whereas at other times, his interictal EEG was profoundly abnormal with hypsarrhythmia. An ictal EEG showed a nonlateralized electrodecremental response at seizure onset. Treatment with 8 different antiepileptic medications had been unsuccessful. The clinical data raised the concern for diffuse occult cortical dysplasia, in addition to the obvious dysplasia in the right temporal lobe. Despite this, resective surgery was still being considered.

MEG source waveforms in this patient were transient and sharp and localized to 4 independent locations: the midportion of the superior temporal gyri bilaterally and the posterior temporal-occipital lobe junctions bilaterally. The activity was always more prominent on the left than on the right (Fig 3).

MEG confirmed the diffuse nature of the abnormality and that the patient was not a surgical candidate, despite the single visible MR imaging lesion in what was presumed to be the nondominant temporal lobe. Surgery with placement of intracranial EEG grids was avoided. Placement of a vagal nerve stimulator (VNS) is being considered by the family. 

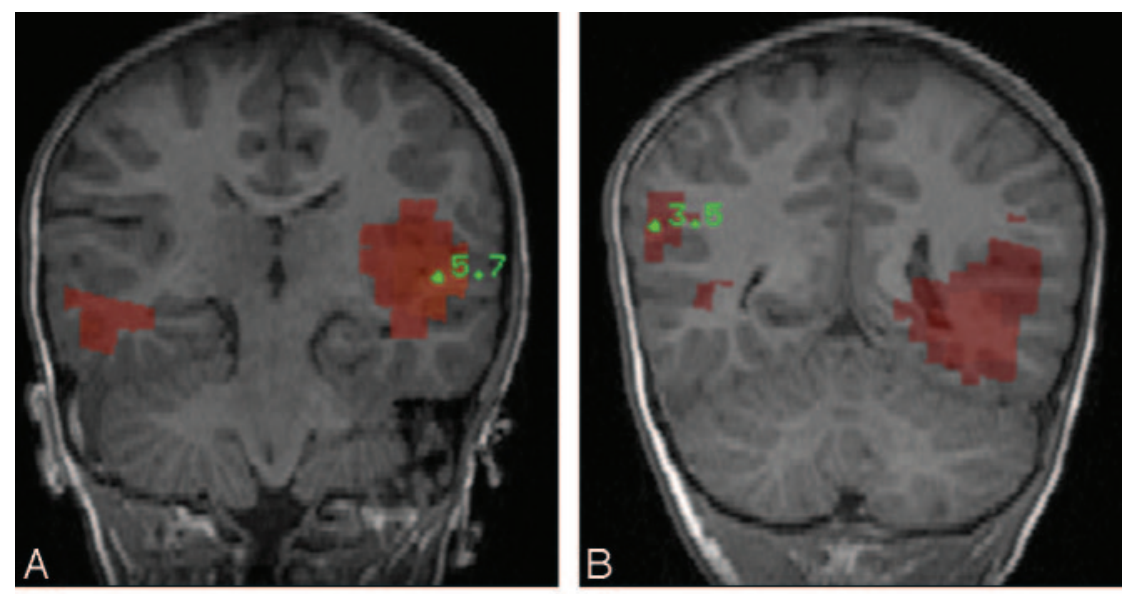

Fig 3. Multifocal Activity. MEG interictal activity is seen as regions of color superimposed over the midportion of the superior temporal gyri bilaterally and the posterior temporaloccipital lobe junctions bilaterally on coronal reformation $(A$ and $B$ ) and axial ( $C$ and $D$ ) MPRAGE MR images.
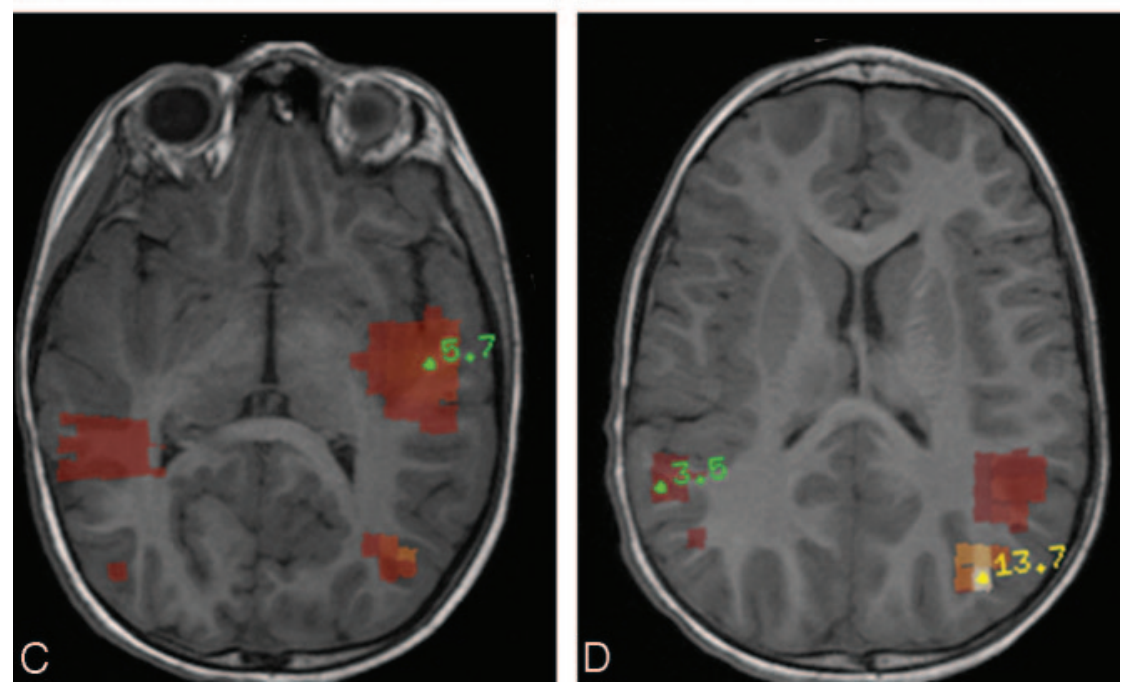

\section{Discussion}

As our experience with MEG grows, we increasingly embrace this new technique. The ability to detect the IOZ and the relationship of the IOZ to eloquent cortex has become a valuable tool in the presurgical evaluation of the pediatric patient with medically refractory epilepsy. As indicated in the on-line Table, the primary clinical roles for MEG have all been encountered with the first 10 patients examined.

Specifically, MEG can detect the IOZ when the MR imaging and other noninvasive imaging methods have failed to localize the lesion. Even with submillimeter volumetric sequences on a $3 \mathrm{~T}$ clinical imaging system with image interpretation by experienced pediatric neuroradiologists, the structural abnormality will not always be detected, as in our cases 2 , 5,6 , and 10 .

Previously, children such as these would typically not have been considered surgical candidates. Many would have been implanted with VNSs, which currently preclude subsequent imaging at magnetic fields above $1.5 \mathrm{~T}$ and with systems that do not have a dedicated transmit-receive head coil. Although our early experience with performing MEG in the presence of a VNS was unsuccessful (case 9), a subsequent attempt with another patient was successful. In keeping with the current literature, though the single equivalent dipole analysis method is always nondiagnostic due to artifact from the VNS, new data indicate that SAMg2 analysis can be successfully performed in a percentage of patients. ${ }^{42}$ Other more sophisticated source- modeling approaches also appear promising for reduction of VNS-related artifact. ${ }^{43}$ Because VNS results in seizure reduction of $\geq 50 \%$ in one third of patients and a reduction in seizure frequency of $30 \%-50 \%$ in one third of patients, ${ }^{44}$ our hope is that these children who were not previously considered candidates for surgical resection may now have hope that a surgically amenable lesion can be found.

Also MEG can demonstrate a focal IOZ when seizures are thought to be multifocal due to rapid propagation. As in our first case example, extremely rapid seizure spread between frontal lobes can be difficult to detect with scalp EEG. MEG can be particularly valuable in this setting. ${ }^{45-47}$ In both sensor space and source space, the "lead-in" from the IOZ and the secondary involvement of connected brain regions often can be discovered.

Conversely, MEG can find multifocal independent IOZ, making successful resective surgery and seizure-freedom unlikely. Like our second case example (on-line Table, case 7), having a single focus identified with MR imaging does not guarantee a single IOZ. As with the patient without an identifiable lesion on MR imaging, finding a single lesion should not mistakenly create "satisfaction of search." We currently consider performing MEG on every patient with epilepsy during the presurgical evaluation.

In the setting of epilepsy, MEG can provide confirmatory information when the positron-emission tomography, MR imaging, scalp EEG, and seizure semiology are concordant. 
This allows the neurosurgeon, neurologist, patient, and family a higher degree of confidence that the IOZ has been accurately identified, potentially obviating the phase II evaluation. Eliminating the need for 2 surgical procedures reduces the length of hospitalization and the risk of complications associated with the administration of GA as well as those associated with subdural grid placement, including infection (either primary or reactivation), ischemia, CSF leak, and hemorrhage, occasionally requiring transfusion. ${ }^{48-50}$ Additional potential benefits include decreased surgical and inpatient hospitalization costs, fewer lost work days for caregivers, and improving the overall experience for the family.

Finally, the relationship of the IOZ to eloquent brain can be accurately determined. The importance of this cannot be understated. Prolonged phase II evaluation may be avoided when the IOZ is in the primary motor cortex or in the known regions responsible for receptive or expressive language function. When this scenario is encountered, the patient and family can be prepared for the likelihood that the IOZ cannot be safely resected and discussions of the value of placement of a VNS and/or creation of multiple subpial transections can take place preoperatively. Similarly, the trajectories created from the DTI data show the relationship of the IOZ to important white matter tracts, particularly the ipsilateral corticospinal tract, potentially altering the surgical approach.

\section{Conclusions}

Our experience with MEG in the complex heterogeneous patient population seen at our tertiary referral center has been overwhelmingly positive. The MEG findings have impacted patient management in nearly every case and have been universally accepted by our pediatric neurologists and pediatric neurosurgeon as beneficial for their patients. Specifically, these benefits include substantiated decisions not to offer surgical resection; decisions to move ahead with intracranial grid placement and surgical resection, including guiding electrode placements; and lesion detection in the setting of nonlesional structural imaging. As we gain more experience with this emerging technique at our institution, we anticipate further significant influence on patient management.

\section{Acknowledgments}

We thank the following individuals for their contributions to the constructive discussions of patient management and for referring their patients for MEG examination: Robert Clancy, MD; Brenda Porter, MD; Susan Melamed, CRNP; Joseph Sullivan, MD; Lawrence Brown, MD; Amy Brooks-Kayal, MD; Christina Bergqvist, MD; and Jeffrey Golden, MD. We also thank Neeti Suri, MD, for assistance with MR trajectory.

\section{References}

1. Holden EW, Thanh Nguyen H, Grossman E, et al. Estimating prevalence, incidence, and disease-related mortality for patients with epilepsy in managed care organizations. Epilepsia 2005;46:311-19

2. Morgan CL, Kerr MP. Epilepsy and mortality: a record linkage study in a U.K. population. Epilepsia 2002;43:1251-55

3. Helmstaedter C, Kurthen M, Lux S, et al. Chronic epilepsy and cognition: a longitudinal study in temporal lobe epilepsy. Ann Neurol 2003;54:425-32

4. Piazzini A, Turner K, Chifari R, et al. Attention and psychomotor speed decline in patients with temporal lobe epilepsy: a longitudinal study. Epilepsy Res 2006;72:89-96. Epub 2006 Aug 22
5. Jokeit H, Ebner A. Effects of chronic epilepsy on intellectual functions. Prog Brain Res 2002;135:455-63

6. Gleissner U, Sassen R, Schramm J, et al. Greater functional recovery after temporal lobe epilepsy surgery in children. Brain 2005;128(Pt 12):2822-29. Epub 2005 Jul 13

7. Helmstaedter C, Elger CE. Functional plasticity after left anterior temporal lobectomy: reconstitution and compensation of verbal memory functions. Epilepsia 1998;39:399-406

8. Wyllie E, Comair YG, Kotagal P, et al. Seizure outcome after epilepsy surgery in children and adolescents. Ann Neurol 1998;44:740-48

9. Pataraia E, Simos PG, Castillo EM, et al. Does magnetoencephalography add to scalp video-EEG as a diagnostic tool in epilepsy surgery? Neurology 2004;62:943-48

10. Grondin $\mathrm{R}$, Chuang $\mathrm{S}$, Otsubo $\mathrm{H}$, et al. The role of magnetoencephalography in pediatric epilepsy surgery. Childs Nerv Syst 2006;22:779-85. Epub 2006 Jun 28

11. Papanicolaou AC, Pataraia E, Billingsley-Marshall R, et al. Toward the substitution of invasive electroencephalography in epilepsy surgery. J Clin Neurophysiol 2005;22:231-37

12. Fischer MJ, Scheler G, Stefan H. Utilization of magnetoencephalography results to obtain favourable outcomes in epilepsy surgery. Brain 2005;128(Pt 1):153-57. Epub 2004 Nov 24

13. Otsubo H, Chitoku S, Ochi A, et al. Malignant rolandic-sylvian epilepsy in children: diagnosis, treatment, and outcomes. Neurology 2001;57:590-96

14. Stefan H, Hummel C, Scheler G, et al. Magnetic brain source imaging of focal epileptic activity: a synopsis of 455 cases. Brain 2003;126(Pt 11):2396-405. Epub 2003 Jul 22

15. Wu JY, Sutherling WW, Koh S, et al. Magnetic source imaging localizes epileptogenic zone in children with tuberous sclerosis complex. Neurology 2006;66:1270-72

16. Shibasaki H, Ikeda A, Nagamine T. Use of magnetoencephalography in the presurgical evaluation of epilepsy patients. Clin Neurophysiol 2007;118: 1438-48. Epub 2007 Apr 23

17. Rampp S, Stefan H. Magnetoencephalography in presurgical epilepsy diagnosis. Expert Rev Med Devices 2007;4:335-47

18. Knake S, Halgren E, Sheraishi H, et al. The value of multichannel MEG and EEG in the presurgical evaluation of 70 epilepsy patients. Epilepsy Res 2006;69: 80-86. Epub 2006 Mar 3

19. Ghatan S. Surgery for epilepsy. Pediatr Ann 2006;35:386-93

20. RamachandranNair R, Otsubo H, Shroff MM, et al. MEG predicts outcome following surgery for intractable epilepsy in children with normal or nonfocal MRI findings. Epilepsia 2007;48:149-57

21. Mohamed IS, Otsubo H, Donner E, et al. Magnetoencephalography for surgical treatment of refractory status epilepticus. Acta Neurol Scand Suppl 2007;115:29-36

22. Wennberg R. Magnetic source imaging versus intracranial electroencephalogram: neocortical versus temporolimbic epilepsy surgery. Ann Neurol 2006;60:271

23. Knowlton RC. The role of FDG-PET, ictal SPECT, and MEG in the epilepsy surgery evaluation. Epilepsy Behav 2006;8:91-101

24. Shih JJ, Weisend MP, Lewine J, et al. Areas of interictal spiking are associated with metabolic dysfunction in MRI-negative temporal lobe epilepsy. Epilepsia 2004;45:223-29

25. Oishi M, Kameyama S, Masuda H, et al. Single and multiple clusters of magnetoencephalographic dipoles in neocortical epilepsy: significance in characterizing the epileptogenic zone. Epilepsia 2006;47:355-64

26. Kamimura T, Tohyama J, Oishi M, et al. Magnetoencephalography in patients with tuberous sclerosis and localization-related epilepsy. Epilepsia 2006;47:991-97

27. Jansen FE, Huiskamp G, van Huffelen AC, et al. Identification of the epileptogenic tuber in patients with tuberous sclerosis: a comparison of high-resolution EEG and MEG. Epilepsia 2006;47:108-14

28. Shiraishi H, Ahlfors SP, Stufflebeam SM, et al. Application of magnetoencephalography in epilepsy patients with widespread spike or slow-wave activity. Epilepsia 2005;46:1264-72

29. Bast T, Ramantani F, Boppel T, et al. Source analysis of interictal spikes in polymicrogyria: loss of relevant cortical fissures requires simultaneous EEG to avoid MEG misinterpretation. Neuroimage 2005;25:1232-41

30. Smith MC. The utility of magnetoencephalography in the evaluation of secondary bilateral synchrony: a case report. Epilepsia 2004;45(suppl 4):57-60

31. Baumgartner C. Controversies in clinical neurophysiology: MEG is superior to EEG in the localization of interictal epileptiform activity-Con. $\mathrm{Clin} \mathrm{Neu}$ rophysiol 2004;115:1010-20

32. Balakrishnan $\mathrm{G}$, Grover KM, Mason $\mathrm{K}$, et al. A retrospective analysis of the effect of general anesthetics on the successful detection of interictal epileptiform activity in magnetoencephalography. Anesth Analg 2007;104:1493-97, table of contents

33. Szmuk P, Kee S, Pivalizza EB, et al. Anaesthesia for magnetoencephalography in children with intractable seizures. Paediatr Anaesth 2003;13:811-17

34. Kirsch HE, Mantle M, Nagarajan SS. Concordance between routine interictal magnetoencephalography and simultaneous scalp electroencephalography in a sample of patients with epilepsy. J Clin Neurophysiol 2007;24:215-31 
35. Lin YY, Shih YH, Hsieh JC, et al. Magnetoencephalographic yield of interictal spikes in temporal lobe epilepsy: comparison with scalp EEG recordings. $\mathrm{Neu}$ roimage 2003;19:1115-26

36. Bast T, Wright T, Boor R, et al. Combined EEG and MEG analysis of early somatosensory evoked activity in children and adolescents with focal epilepsies. Clin Neurophysiol 2007;118:1721-35

37. Robinson SE, Nagarajan SS, Mantle M, et al. Localization of interictal spikes using SAM(g2) and dipole fit. Neurol Clin Neurophysiol 2004;Nov 30:74

38. Xiang J, Holowka S, Qiao H, et al. Automatic localization of epileptic zones using magnetoencephalography. Neurol Clin Neurophysiol 2004;Nov 30:98.

39. Hirata M, Kato A, Taniguchi M, et al. Determination of language dominance with synthetic aperture magnetometry: comparison with the Wada test. $\mathrm{NeU}$ roimage 2004;23:46-53

40. Mori S, Crain BJ, Chacko VP, et al. Three-dimensional tracking of axonal projections in the brain by magnetic resonance imaging. Ann Neurol 1999;45:265-69

41. Jiang, H, van Zijl PC, Kim S, et al. DtiStudio: resource program for diffusion tensor computation and fiber bundle tracking. Comput Methods Programs Biomed 2006;81:106-16. Epub 2006 Jan 18

42. Rose DF, Fujiwara H, Hemasilpin N, et al. Application of beamformer algorithm to MEG spontaneous recordings in epilepsy patients with vagal nerve stimulators. Presented at: First Conference of International Society for the Advancement of Clinical Magnetoencephalography, Matsushima, Japan, August $27-30,2007$
43. Taulu S, Simola J. Spatiotemporal signal space separation method for rejecting nearby interference in MEG measurements. Phys Med Biol 2006;51:1759-68

44. Boon $\mathrm{P}$, Herdt $\mathrm{V}$, Vonck $\mathrm{K}$, et al. Clinical experience with vagus nerve stimulation and deep brain stimulation in epilepsy. Acta Neurochir Suppl 2007;97(Pt 2):273-80

45. Hara K, Lin FH, Camposano S, et al. Magnetoencephalographic mapping of interictal spike propagation: a technical and clinical report. AJNR Am J Neuroradiol 2007;28:1486-88

46. Tanaka N, Kamada K, Takeuchi F, et al. Magnetoencephalographic analysis of secondary bilateral synchrony. J Neuroimaging 2005;15:89-91

47. Ossenblok P, de Munck JC, Colon A, et al. Magnetoencephalography is more successful for screening and localizing frontal lobe epilepsy than electroencephalography. Epilepsia 2007;48:2139-49. Epub 2007 Jul 28

48. Johnston JM Jr, Mangano FT, Ojemann JG, et al. Complications of invasive subdural electrode monitoring at St. Louis Children's Hospital, 1994-2005. J Neurosurg 2006;105(suppl 5):343-47

49. Musleh W, Yassari R, Hecox K, et al. Low incidence of subdural grid-related complications in prolonged pediatric EEG monitoring. Pediatr Neurosurg 2006; $42: 284-87$

50. Onal C, Otsubo H, Araki T, et al. Complications of invasive subdural grid monitoring in children with epilepsy. J Neurosurg 2003;98:1017-26 